Original Paper http://ajol.info/index.php/ijbcs http://indexmedicus.afro.who.int

\title{
Dimensionnement, fonctionnement et exploitation des Lits de Séchages à Ecoulement Non Saturé (LSENS) pour le traitement des boues de vidange des fosses septiques
}

\author{
Théophile GNAGNE ${ }^{1,2 *}$, Yao Francis KOUAME ${ }^{2,3}$ et Tenena Martial YEO ${ }^{1,2}$ \\ ${ }^{1}$ Laboratoire Géoscience et Environnement, UFR des Sciences et Gestion de l'Environnement, Université \\ Nangui Abrogoua, 02 BP 801 Abidjan 02, Côte d'Ivoire. \\ ${ }^{2}$ Agence Panafricaine Intergouvernementale Eau et Assainissement pour l'Afrique Représentation Nationale de \\ Côte d'Ivoire, 18 BP 80 Abidjan 18, Côte d'Ivoire. \\ ${ }^{3}$ Laboratoire des Sciences et Technologie de l'Environnement, UFR des Sciences et Gestion de \\ l'Environnement, Université Jean Lorougnon Guédé de Daloa BP 150 Daloa, Côte d'Ivoire. \\ *Auteur correspondant ; E-mail : gnagne.theophile@gmail.com; Tél. : 0022579037525.
}

\section{RESUME}

Les résultats de l'échelle laboratoire montrent de bonnes performances épuratoires des boues de vidange des fosses septiques par la technologie du Lit de Séchage à Ecoulement Non Saturé (LSENS). En fait, le LSENS est un de Lit de Séchage Traditionnel (LST) au sein duquel l'eau et l'air riche en oxygène s'écoulent grâce à son fonctionnement fait de courtes périodes $(30 \mathrm{mn}$ ) d'alimentation et de longues périodes (3jours) de séchage. Cet article montre, à travers des essais réalisés sur un pilote de $80 \mathrm{~m}^{2}$, que les bonnes capacités épuratoires obtenues sur les colonnes $0,46 \mathrm{~m}^{2}$ sont transférables. Une charge organique moyenne de 0,34 $\mathrm{kg} / \mathrm{j} / \mathrm{m}^{2}$ a été appliquée sur le pilote avec des boues caractérisées par une DCO de $4050 \mathrm{mgO}_{2} / \mathrm{L}, \mathrm{NTK}$ $990 \mathrm{mgN} / \mathrm{L}$ et CF $\left(2,8 \cdot 10^{7} \mathrm{UFC} / 100 \mathrm{~mL}\right)$. A cette charge, la DCO et le NTK sont éliminés à plus de $95 \%$ avec un abattement de 4 Ulog de CF. Ces résultats sont comparables à ceux obtenus avec les essais sur colonnes de laboratoire. Ainsi, le passage des capacités épuratoires du LSENS de l'échelle réduite (laboratoire) à une échelle beaucoup plus grande (pilote) est établi. Cela ouvre une perspective du recours au LSENS comme alternative crédible pour le traitement des boues de vidange à l'échelle d'une ville en milieu tropical.

(C) 2019 International Formulae Group. All rights reserved

Mots clés : abattement bactérien, fractionnement des apports, milieu poreux, surface d'infiltration

\section{Design, operation and maintenance of Unsaturated Flow Drying Bed (UFDB) for septic tanks sludge treatment}

\begin{abstract}
The results of lab scale work show good purification performance of the Unsaturated Flow Drying Bed (UFDB) for the treatment of septic tank sludge. Indeed, the UFDB is a Traditional Drying Bed (TDB) in which
\end{abstract}


water and oxygen-rich air flow through its operation for short periods (30 minutes) of feeding and long drying time ( 3 days). This article shows, through tests carried out on a pilot of $80 \mathrm{~m}^{2}$, that the good purification capacities obtained on the columns of $0.46 \mathrm{~m}^{2}$ are transferable. An average organic load of $0.34 \mathrm{~kg} / \mathrm{d} / \mathrm{m}^{2}$ was applied to the pilot with sludge characterized by a COD of $4050 \mathrm{mgO}_{2} / \mathrm{L}$, TKN $990 \mathrm{mg} / \mathrm{L}$ and faecal coliforms $\left(2.8 \times 10^{7} \mathrm{CFU} / 100 \mathrm{~mL}\right)$. At this load, COD and TKN are eliminated over $95 \%$ with a reduction of 4 Ulog of faecal coliforms. These results are comparable to those obtained with laboratory column tests. Thus, the passage of UFDB purification performance from the reduced scale (laboratory) to a much larger scale (pilot) is established. This opens up the use of the Unsaturated Flow Drying Bed as a credible alternative for the treatment of fecal sludge at the scale of a city in a tropical environment.

(C) 2019 International Formulae Group. All rights reserved

Keywords: bacterial abatement, supplies splitting, porous area, infiltration surface

\section{INTRODUCTION}

Dans les villes africaines la majorité des populations font recours à l'assainissement autonome des eaux usées. Cette approche, pourtant convenable pour ces villes, est mal mise en œuvre, souffrant de l'insuffisance de certains compartiments de la chaine de l'assainissement. En effet, les fosses septiques ne sont toujours pas équipées de puits d'infiltration. Pire, lorsque que les fosses sont pleines, les boues sont, soit manuellement vidangées et enfouies in situ ou vidangées par des camions mais rejetées dans le milieu naturel sans traitement (Agnès et Martin, 2002 ; CREPA, 2004).

Pourtant, l'assainissement autonome est un choix technologique crédible en application du principe de traitement décentralisé des déchets. Pour qu'il en soit ainsi, les puits d'infiltration convenablement exécutés assurent une partie du traitement in situ, la vidange des boues convenablement assurée remplace le réseau d'égouts et une station de traitement des boues achève le traitement avant rejet ou valorisation. Traditionnellement, le traitement se fait à travers les lits de séchage qui servent de prétraitement (Koné et Strauss, 2004).

Une amélioration du lit de séchage traditionnel peut provenir du fractionnement des apports de boues de vidanges. La quantité de boues appliquées au cours d'une séquence d'alimentation, suivie d'une période de repos, est de l'ordre d'un centimètre d'épaisseur.
Dans ces conditions, les périodes de séchage sont courtes, soit deux à trois jours seulement pour le séchage des matières en suspensions (MES) retenues à la surface d'infiltration. Autrement dit, l'application d'une faible épaisseur de boues permet de profiter pleinement du fort ensoleillement des pays tropicaux pour sécher rapidement les MES retenues à la surface du lit (Kouamé et al., 2010 ; Gnagne et al., 2014).

$\mathrm{Ce}$ mode d'alimentation induit l'écoulement de fractions liquides dans le massif poreux en milieu non saturé qui entraine la circulation de l'air. Cela permet des apports d'oxygène qui, contrairement à ce qui se passe dans les lits de séchage traditionnels, favorisent la dégradation des pollutions carbonées et azotées par oxydation ainsi que la pollution microbienne. Cette technologie est baptisée : Lits de Séchage à Ecoulement Non Saturé (LSENS).

Cette approche s'apparente à l'épuration par infiltration utilisée avec succès au Maroc, en France, en Espagne et au Burkina Faso. En effet, l'alternance de phases d'alimentation et de repos permet d'épurer efficacement les eaux usées avec un bon abattement bactérien allant jusqu'à 5 ULog (Bancolé, 2001; Gnagne, 2003).

En fait, le LSENS est un procédé hybride, qui tient compte à la fois des lits de séchage traditionnels et de l'épuration par infiltration intermittente sur sable. Il cumule les avantages de ces deux technologies. De la 
première, il emprunte le séchage naturel facilité par l'ensoleillement sous climat tropical sec ou humide. De la seconde, il emprunte le fractionnement des apports par alternance de phases d'alimentation et de repos qui permet d'épurer efficacement les effluents de forte charge oxydable (Bancolé et al., 2003 ; Coulibaly et al., 2008 ; Kouamé et al., 2008).

Des essais à l'échelle réduite en laboratoire donnent des résultats satisfaisants. En effet, avec une charge polluante traitable de $0,34 \mathrm{~kg} / \mathrm{j} / \mathrm{m}^{2}$, les rendements d'élimination sur les colonnes de laboratoire sont de $96 \%$, $97,5 \%$ et $99 \%$ respectivement pour la DCO, l'azote total et la turbidité. A ces rendements, les valeurs moyennes de DCO, d'azote total et de la turbidité des filtrats restitués par les colonnes sont respectivement $46 \mathrm{mgO}_{2} / \mathrm{L}, 20$ $\mathrm{mg} / \mathrm{L}$ et 10,5 UNT (Gnagne et al., 2014).

Il reste la question du passage à une échelle beaucoup plus grande (pilote) avant l'application du LSENS pour le traitement des boues de vidange des villes sous climat tropicale. L'objectif de cet article est de montrer que les performances du LSENS constatées à l'échelle réduites peuvent être transférées à l'échelle pilote.

\section{MATERIEL ET METHODES Matériel}

Le pilote du LSENS est un lit de séchage avec une surface de $80 \mathrm{~m}^{2}$. Il est de forme rectangulaire de longueur $16,5 \mathrm{~m}$, de largeur $5,50 \mathrm{~m}$ et de profondeur $1,50 \mathrm{~m}(1 \mathrm{~m}$ enterré et $0,5 \mathrm{~m}$ à la surface). Il est compartimenté en deux unités d'infiltration (casiers) de $40 \mathrm{~m}^{2}$ alimentés en alternance. Des drains d'aération sont placés à $35 \mathrm{~cm}$ de profondeur et espacés de $50 \mathrm{~cm}$. Le fond du lit est aménagé sous une forme incurvée vers le bas avec une pente de $6 \%$ de sorte à collecter et à drainer le filtrat traité par l'intermédiaire d'une conduite crépinée (Figure 1).

Le massif épurateur est du sable son épaisseur dans le lit est de $1 \mathrm{~m}$. Le massif drainant est constitué de graviers de diamètre compris entre $20 \mathrm{~mm}$ et $40 \mathrm{~mm}$. Son épaisseur est de $0,2 \mathrm{~m}$. Ceci donne un total de $1,2 \mathrm{~m}$ d'épaisseur de matériau filtrant.

\section{Dimensionnement \\ Calcul}

Pour obtenir une répartition relativement homogène sur la surface d'infiltration, il faut une bâchée de $0,1 \mathrm{~m}$ de boues avec un temps de séchage de 72 heures avant l'admission d'une nouvelle bâchée dans les régions à fort ensoleillement comme les zones tropicales sèches. Dans ce cas, il est réalisé des casiers dont la surface d'infiltration est en adéquation avec les capacités des camions de vidange pour respecter les $0,1 \mathrm{~m}$ de boue à chaque dépotage. A titre d'exemple, pour une ville dont la majorité des camions de vidange a une capacité $\operatorname{deV}_{\mathrm{c}}\left(\mathrm{m}^{3}\right)$, la surface d'infiltration du casier ou surface d'infiltration élémentaire $\left(\mathrm{S}_{\text {infe }}\right)$ est :

$\mathrm{S}_{\text {infe }}=\frac{V c}{0,1}$

De même, si la ville produit un volume journalier $V_{b}$ de boues, l'aire d'infiltration totale $\left(\mathrm{A}_{\text {inf }}\right)$ de la station vaut :

$\mathrm{A}_{\text {inf }}=N j * \frac{V b}{0,1}$

Avec $\mathrm{N}_{\mathrm{j}}$ le nombre de jours de repos.

Le nombre total de casier $\left(\mathrm{N}_{\mathrm{c}}\right)$ du LSENS est :

$\mathrm{N}_{\mathrm{c}}=\frac{\operatorname{Ainf}}{\operatorname{Sinf}}$

Le nombre de casier doit être au moins égal à 3.

En général, pour réaliser une restauration totale par la mise au repos des casiers pendant 3 mois, il faut réaliser $15 \%$ de casiers en plus.

Il faut adopter une hauteur de $1 \mathrm{~m}$ de sable comme épaisseur du lit constitué d'un sable moyen à granulométrie serrée, caractérisée par un diamètre moyen $(\mathrm{Dm}=420 \mu \mathrm{m}$;) et un coefficient d'uniformité $\mathrm{CU}=2,5$.

La charge polluante traitable est égale à la variation de la Demande Totale en 
Oxygène DTO $($ DTO $=$ DCO $+4,57$ x NTK en $\mathrm{kg} / \mathrm{m}^{3}$ ) multipliée par la charge hydraulique moyenne journalière $(\mathrm{m} / \mathrm{j})$.

\section{Dispositions constructives}

Le schéma de construction d'un lit de séchage à écoulement non saturé est le même que celui du lit de séchage traditionnel une fois la surface d'infiltration calculée avec le volume moyen de camion de vidange de la ville.

\section{Fonctionnement}

Le camion-citerne dépote tout son contenu sur la surface d'infiltration d'un des casiers du LSENS en 10 minutes en moyenne. Dès lors, le casier qui vient de recevoir la boue est mis au repos pendant 3 jours (72 h).Le dépotage suivant se fait alors dans un autre casier qui sera aussi mis au repos à son tour pendant 3 jours. Au troisième jour, le casier qui avait initialement reçu la boue, qui entre temps a séché et a été raclé, peut à nouveau recevoir le dépôt de boues de vidange.

A travers les successions d'un jour de dépotage et de 3 jours de repos, le massif filtrant du casier se colmate peu à peu. Pour y remédier, il a été établi qu'après 9 mois de dépotage, il faut laisser au repos le casier pendant 1 mois pour une restitution quasitotale de la porosité du massif filtrant.

\section{Exploitation}

L'exploitation de la station requiert la présence d'un personnel un peu qualifié pour guider les vidangeurs à l'effet d'éviter de dépoter avant le terme des jours de repos. Il assure également le raclage de la surface d'infiltration. Le raclage doit être effectué pour faciliter la pénétration par diffusion de l'air dans le massif poreux à travers la surface d'infiltration.

Les analyses de laboratoires sont effectuées selon AFNOR (1994); Rodier (1996); CEAEQ (1999).

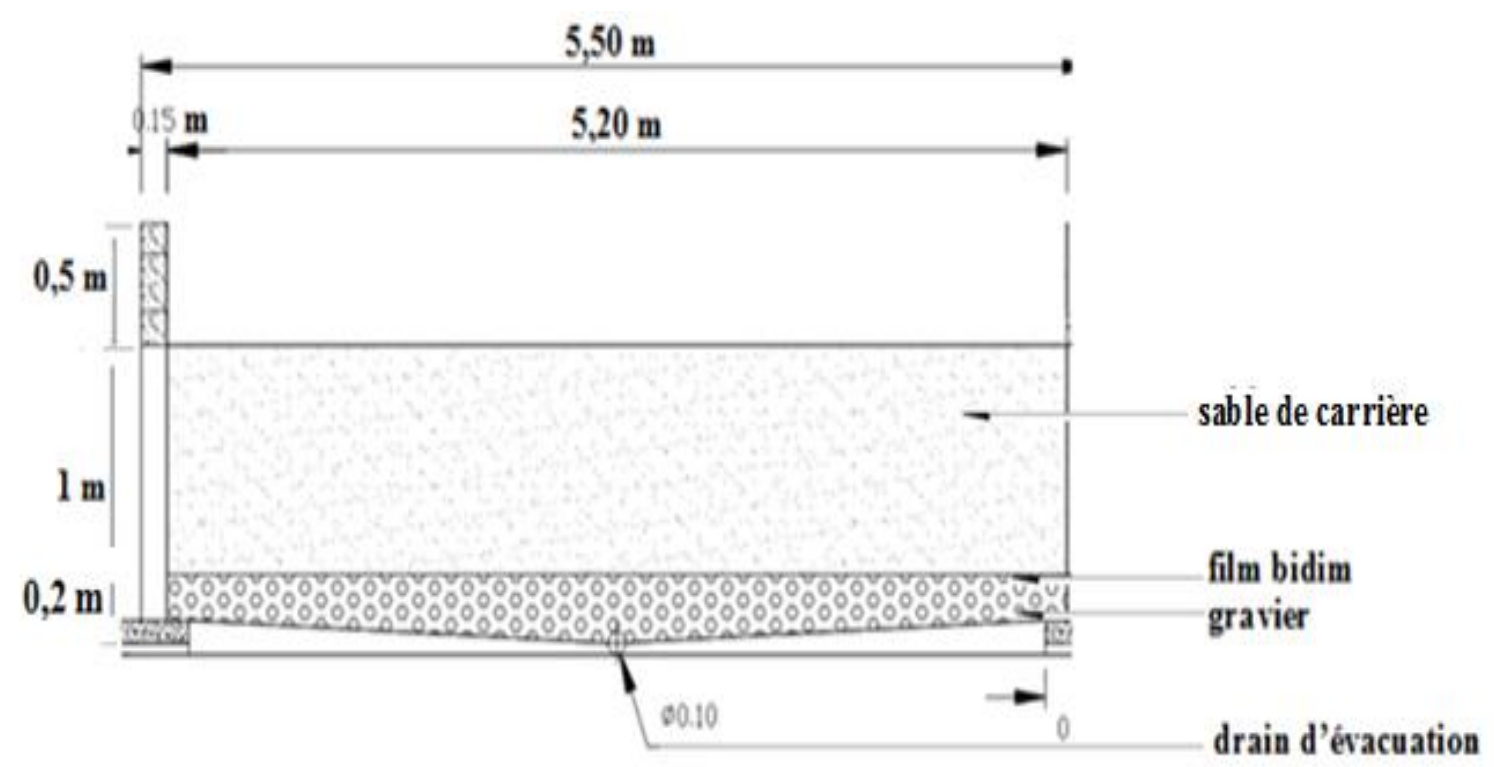

Figure 1: Représentation schématique du LSENS pilote vue de travers. 


\section{RESULTATS}

\section{Infiltration et séchage de la surface du lit}

La Figure 2 montre le processus d'infiltration, de séchage et de raclage des boues sur la surface d'infiltration après l'alimentation d'un casier du LSENS. Le temps d'infiltration varie de 25 minutes à 3 heures avec une moyenne de 1 heure 30 minutes. De façon générale, après 48 heures, le séchage est suffisant pour opérer un raclage permettant de libérer la surface d'infiltration pour l'oxygénation du massif filtrant. La durée du raclage pour une personne, varie de 20 à 40 minutes avec pour valeur moyenne 30 minutes pour deux casiers. La surface ainsi libérée par raclage peut être soumise à une nouvelle alimentation. Les boues raclées sont exposées au soleil en dehors du lit de séchage.

\section{Capacité épuratoire du lit de séchage}

Le Tableau 1 présente des rendements d'élimination de la DCO du NTK ainsi que l'abattement des Coliformes fécaux. Les résultats sont issus du lit de séchage qui a fonctionné pendant 2,5 mois en régimes d'écoulement non saturé avec une charge polluante traitable de $0,34 \mathrm{~kg} / \mathrm{j} / \mathrm{m}^{2}$.

La capacité d'élimination de la pollution par le LSENS se caractérise par une réduction respective de la $\mathrm{DCO}$, de l'azote et de la turbidité de $97 \%, 95 \%$ et $95 \%$. Deux tendances sont observées dans l'évolution des paramètres mesurés des eaux traitées. La première d'octobre à novembre, est relativement stable. La DCO, le NTK, le $\mathrm{NO}_{3}{ }^{-}$ et le Turbidité se situent respectivement entre les valeurs 50 et $100 \mathrm{mgO}_{2} / \mathrm{L} ; 20$ et $60 \mathrm{mg} / \mathrm{L}$; 10 et $30 \mathrm{mg} / \mathrm{L}$ puis 30 et $60 \mathrm{UNT}$. La seconde, de décembre à janvier, les valeurs en sortie fluctuent. $\mathrm{La} \mathrm{DCO}$, le NTK, le $\mathrm{NO}_{3}^{-}$et le Turbidité évoluent respectivement entre les valeurs 130 et $200 \mathrm{mgO}_{2} / \mathrm{L} ; 30$ et $80 \mathrm{mg} / \mathrm{L}$; 15 et $60 \mathrm{mg} / \mathrm{L}$ puis 70 et $170 \mathrm{UNT}$.

La transparence des filtrats se dégrade et atteint 169 UNT trois semaines après la mise en fonctionnement de la station. Elle s'établie au fil des alimentations suite à la réduction de la charge traitable (Figure 3). En effet, cette figure présente une clarification des boues traitées en cohérence avec les rendements épuratoires du Tableau 1. Au départ, il a été observé 2 jours entre deux alimentations avec une dégradation de la clarté de l'eau traitée. Il a alors été adopté d'observer trois jours entre deux alimentations avec une amélioration de la clarté de l'eau traitée.
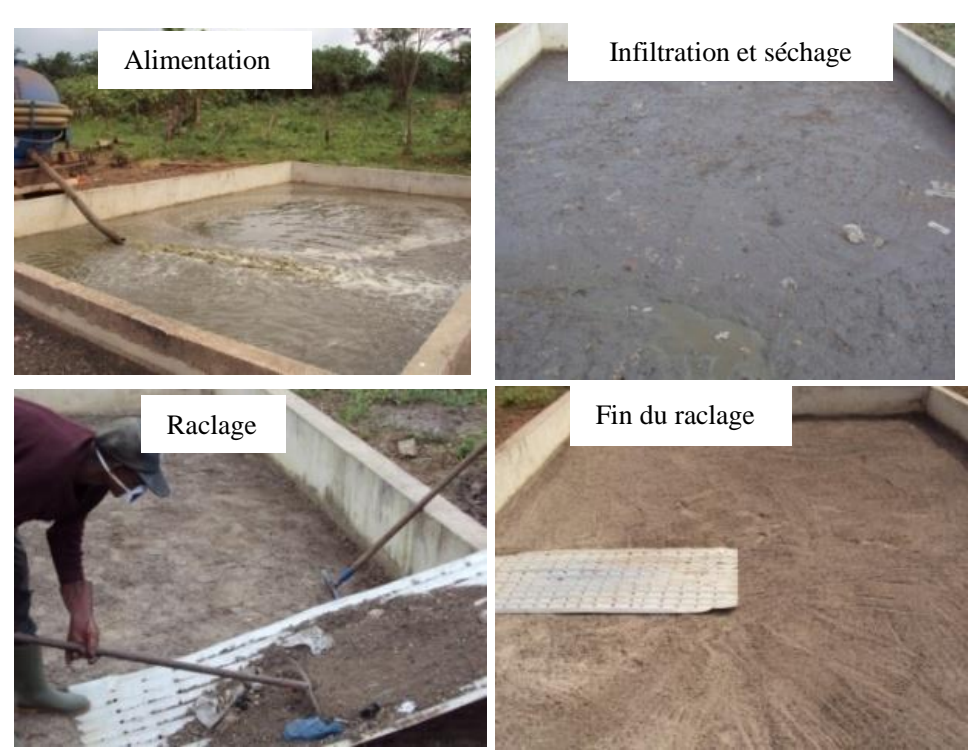
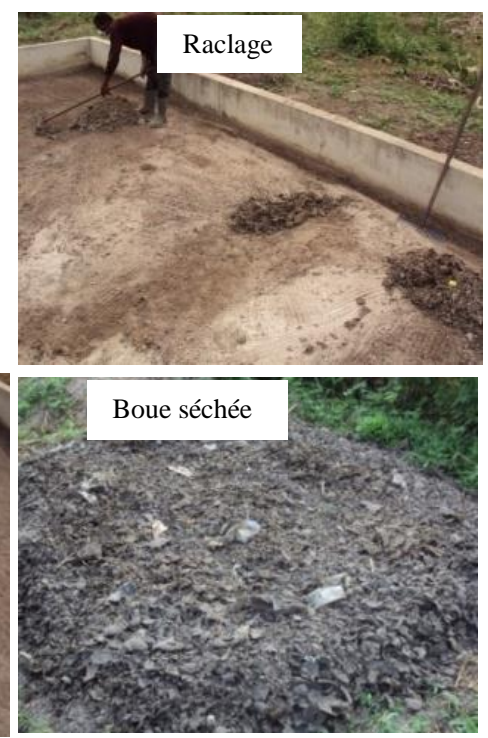

Figure 2 : Infiltration, séchage et raclage du LSENS après alimentation. 
Tableau 1: Caractéristiques physico-chimiqueet bactériologique des boues de vidange brutes (BVB) et traitées (BVT).

\begin{tabular}{|c|c|c|c|c|c|c|c|c|c|c|c|c|c|c|}
\hline \multirow[b]{2}{*}{ Date } & \multicolumn{3}{|c|}{ Turbidité (UNT) } & \multicolumn{3}{|c|}{$\mathrm{DCO}\left(\mathrm{mgO}_{2} / \mathrm{L}\right)$} & \multicolumn{3}{|c|}{ NTK (mg/L) } & \multicolumn{2}{|c|}{$\mathrm{NO}_{3}^{-}(\mathrm{mg} / \mathrm{L})$} & \multicolumn{3}{|c|}{ CF (UFC/100mL) } \\
\hline & BVB & BVT & $\begin{array}{l}\operatorname{Rdt}( \\
\%)\end{array}$ & BVB & BVT & $\begin{array}{l}\text { Rdt } \\
(\%)\end{array}$ & BVB & BVT & $\begin{array}{l}\mathrm{Rdt} \\
(\%)\end{array}$ & BVB & BVT & BVB & BVT & $\begin{array}{l}\text { Abt } \\
\text { (ULog) }\end{array}$ \\
\hline $20 / 10 / 2009$ & 1334 & 61 & & 2537 & 118 & & 864 & 33 & & 2,45 & 10,36 & $2,10 \mathrm{E}+07$ & $3,90 \mathrm{E}+03$ & \\
\hline $22 / 10 / 2009$ & 1308 & 48 & & 3400 & 101 & & 934 & 47 & & 1,63 & 18,67 & & & \\
\hline $24 / 10 / 2009$ & 1906 & 37 & & 2588 & 89 & & 771 & 21 & & 1,49 & 25,07 & $3,60 \mathrm{E}+07$ & $3,10 \mathrm{E}+03$ & \\
\hline $26 / 10 / 2009$ & 1980 & 50 & & 4008 & 114 & & 1051 & 52 & & 2,20 & 12,81 & & & \\
\hline $27 / 10 / 2009$ & 2028 & 45 & & 3197 & 94 & & 981 & 31 & & 1,36 & 20,17 & & & \\
\hline $26 / 11 / 2009$ & 2192 & 36 & & 2917 & 51 & & 850 & 26 & & 2,18 & 30,32 & $1,70 \mathrm{E}+07$ & $1,70 \mathrm{E}+03$ & \\
\hline $30 / 11 / 2009$ & 1980 & 41 & & 3425 & 58 & & 1039 & 28 & & 2,04 & 20,78 & & & \\
\hline $04 / 12 / 2009$ & 2393 & 169 & & 3171 & 136 & & 952 & 72 & & 1,36 & 60,98 & & & \\
\hline $10 / 12 / 2009$ & 1020 & 112 & 95 & 10782 & 165 & 97 & 1401 & 49 & 95 & 1,60 & 15,67 & $2,30 \mathrm{E}+07$ & $1,80 \mathrm{E}+03$ & 4,1 \\
\hline $15 / 12 / 2009$ & 980 & 128 & & 4027 & 148 & & 1024 & 55 & & 1,70 & 32,17 & & & \\
\hline $18 / 12 / 2009$ & 2009 & 93 & & 3679 & 195 & & 958 & 78 & & 2,40 & 49,40 & & & \\
\hline $22 / 12 / 2009$ & 1952 & 140 & & 3044 & 185 & & 771 & 69 & & 4,70 & 13,62 & $4,60 \mathrm{E}+07$ & $1,90 \mathrm{E}+03$ & \\
\hline $26 / 12 / 2009$ & 2089 & 74 & & 6342 & 152 & & 1261 & 48 & & 2,18 & 32,03 & & & \\
\hline $29 / 12 / 2009$ & 1036 & 100 & & 3457 & 108 & & 873 & 32 & & 1,90 & 40,12 & & & \\
\hline 05/01/2010 & 2081 & 80 & & 4186 & 121 & & 1168 & 45 & & 1,70 & 38,16 & $2,50 \mathrm{E}+07$ & $1,70 \mathrm{E}+03$ & \\
\hline Moyenne & 1753 & 81 & & 4051 & 122 & & 993 & 46 & & 2,10 & 28,00 & $2,80 \mathrm{E}+07$ & $2,30 \mathrm{E}+03$ & \\
\hline Ecartype & 475 & 42 & & 2072 & 42 & & 176 & 18 & & 0,80 & 14,50 & $1,09 \mathrm{E}+07$ & $9,29 \mathrm{E}+02$ & \\
\hline
\end{tabular}

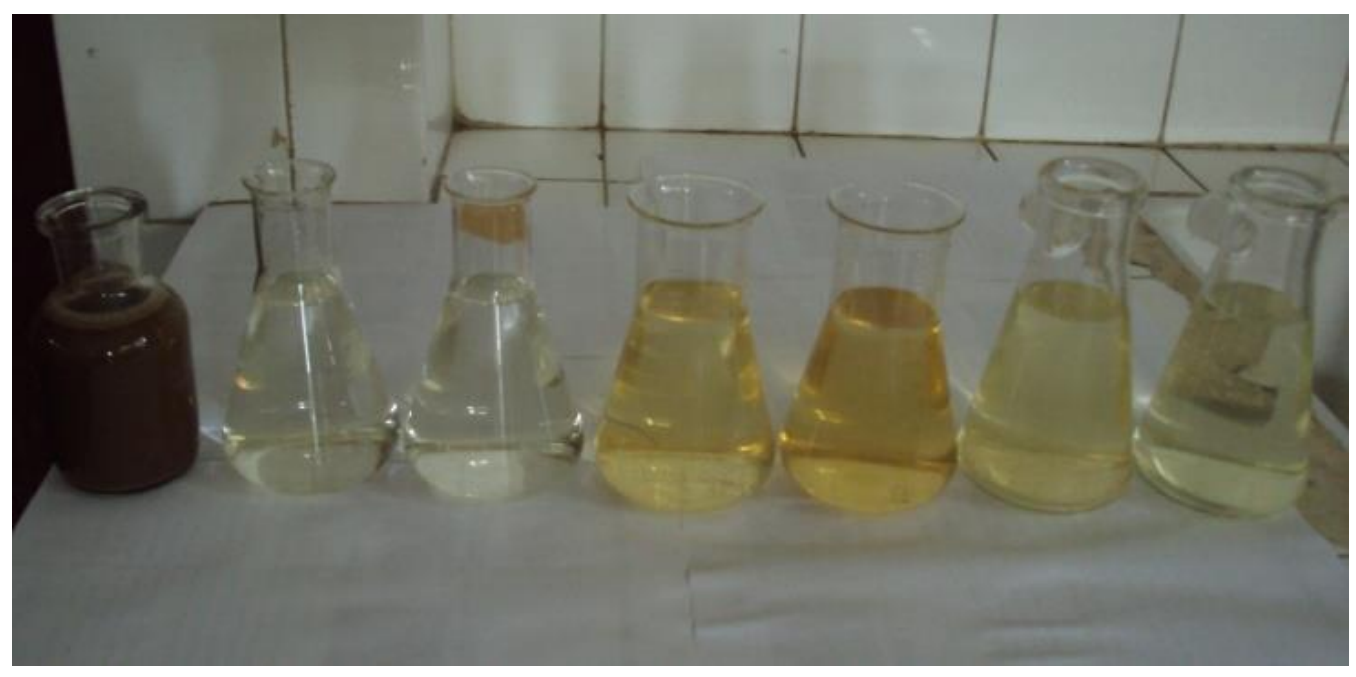

Figure 3 : Evolution de la couleur des boues brutes et des boues traitées durant le fonctionnement du lit de séchage en régime non saturé. 


\section{DISCUSSION}

Le LSENS, issu de l'amélioration du lit de séchage traditionnelle à travers le fractionnement des apports de boues constitue un régime d'écoulement non saturé dans le milieu poreux qu'est le massif filtrant. Ce régime d'écoulement favorise la coexistence d'eau chargée en matière organique et d'air riche en oxygène. Cela débouche sur l'oxydation de la matière organique qui, dans ce cas, permet de traité les boues de vidanges avec des rendements satisfaisant d'élimination de la pollution (Kouamé et al., 2010) comme dans le cas de notre étude. En effet, les rendements d'élimination du LSENS sont de $97 \%, 95 \%$ et $95 \%$ respectivement pour la DCO, l'azote et la turbidité. Des abattements bactériens pouvant aller jusqu'à 4 ULog sont obtenus.

Ainsi, les filtrats issus des apports fractionnés de boues de vidange des fosses septiques sur le LSENS sont conformes aux normes de rejet pour la protection de l'environnement selon l'arrêté $\mathrm{N}^{\circ} 01164 \mathrm{du}$ Ministère de l'Environnement, des Eaux et Forêts (Ahizi, 2008) et utilisable en irrigation selon l'OMS (N'Diaye et al., 2011).

La performance du LSENS par comparaison aux lits de séchage plantés ou non plantés s'explique par son mode de fonctionnement qui consiste au fractionnement des apports de boues et à la réduction des temps de séchage. En effet, du point de vue du fonctionnement des seconds cités, les boues de vidange sont déversées sur la surface d'infiltration jusqu'à atteindre une épaisseur de boue liquide de 30 à $40 \mathrm{~cm}$, voire plus. Selon les conditions climatiques, le temps de séchage des matières en suspensions (MES) issues des boues varie de trois à six semaines (Memento Technique de l'Eau, 1989; Mungray et Kumar, 2008). L'élimination de la Demande Biochimique en Oxygène en 5 jours $\left(\mathrm{DBO}_{5}\right)$ et celle de la Demande Chimique en Oxygène sont respectivement tout au plus de $60 \%$ et $70 \%$ sur lits de séchage non plantés (Koné et Strauss, 2004). De plus, l'élimination des microorganismes reste faible (Agnès et Martin, 2002).

\section{Conclusion}

Les résultats des essais réalisés sur le pilote, d'une surface d'infiltration 174 fois plus grande que celle des colonnes expérimentales montrent une bonne capacité épuratoire du LSENS en grandeur réelle. $\mathrm{La}$ charge organique moyenne de $0,34 \mathrm{~kg} / \mathrm{j} / \mathrm{m}^{2}$ a donné les performances suivantes : la DCO et le NTK sont éliminés à plus de $95 \%$ et un abattement de 4 Ulog de CF. Ainsi, la transférabilité des capacités épuratoires du LSENS de l'échelle réduite (laboratoire) à une échelle beaucoup plus grande (pilote) est établie. Cela ouvre une perspective du recours au LSENS comme alternative crédible pour le traitement des boues de vidange à l'échelle d'une ville en milieu tropical.

\section{CONFLIT D'INTERETS}

Les auteurs ne déclarent aucun conflit d'intérêts en lien avec le présent article.

\section{CONTRIBUTIONS DES AUTEURS}

TG est le Directeur scientifique de cette étude, il a suivi et mieux orienté les objectifs de ces travaux. YFK et TMY ont effectué les sorties de terrains, analyses au laboratoire et ont rédigé le manuscrit.

\section{REMERCIEMENTS}

Ce travail a été réalisé dans le cadre du volet étude du projet intitulé Boue de vidange, piloté par la représentation nationale de l'Agence Panafricaine pour l'Eau et l'Assainissement pour l'Afrique (EAA). Nous voudrions exprimer notre profonde reconnaissance à cette institution et ses différents responsables.

\section{REFERENCES}

AFNOR. 1994. Qualité de l'Eau (1ère édn). AFNOR: Paris ; 862p.

Agnès M, Martin S. 2002. Gestion des boues de vidange. Institut fédéral pour l'aménagement, l'épuration et la protection des eaux, Duebendorf, Suisse, Département Eau et Assainissement dans les pays en développement, EAWAG/SANDEC 60p. 
Ahizi AD. 2008. Arrêté $\mathrm{N}^{\circ}$ 01164/MINEEF/CIAPOL/SDIIC du Ministère de l'Environnement, des eaux et forêts de Côte d'Ivoire portant réglementation des rejets et émissions des installations classées pour la protection de l'environnement, $7 \mathrm{p}$.

Bancolé DA. 2001. L'oxydation en infiltration percolation. Thèse de Doctorat de l'Université des Sciences du Languedoc, Montpellier II, 150p.

Bancole A, Brissaud F, Gnagne T. 2003. Oxidation processes and clogging in intermittent unsaturated infiltration. Water Science Technology, 11(48): 139146.

CEAEQ. 1999. Détermination des solides dissous totaux et volatils dans les effluents : méthode gravimétrique, Centre d'Expertise en Analyse Environnementale du Québec, 9p.

Coulibaly L, Ama AB, Savané I, Babo Y. 2008. Un filtre à sable perforé à alimentation intermittente pour l'épuration des effluents d'huilerie de palme: cas de l'unité industrielle de Toumanguié, Côte D'ivoire. European Journal of Scientific Research, 20(2) : 226-238.

CREPA. 2004. Stratégie de gestion des boues de vidange issues des fosses septiques de la ville de Bouaké puis Abengourou en Côte d'Ivoire. Rapport d'étude du Centre Régional pour l'Eau Potable et Assainissement (CREPA), $29 \mathrm{p}$.

Gnagne T, Brissaud F. 2003. Etude des potentialités d'épuration d'effluent d'abattoir par infiltration sur sable en milieu tropical. Sud Science et Technologie, 11: 4-11.

Gnagne T, Yéo TM, Kouamé YF, Konan KF. 2014. Unsaturated Flow Drying Bed (UFDB): An Alternative for Treatment of Septic Tanks Sludge. Journal of Water Resource and Protection, 6: 454462.

Koné D, Strauss M. 2004. Low-cost options for treating faecalsludges (FS) in developing countries; challenges and performance; Eawag/Sandec. p. 9.

Kouamé YF, Gnagne T, Konan KF, Yéo TM, Houénou PV, Brissaud F. 2008. Choix du matériau poreux pour l'épuration des boues de vidange des fosses septiques par lit de séchage à écoulement non sature (LSENS). Tribune de l'Eau, $\mathbf{6 4 2}$ : $15-24$.

Kouamé YF, Yéo TM, Gnagne T, Konan KF, N'Gouandi KF, Yapo OB, Séka A, Houénou PV. 2010. Stratégie d'élimination de l'azote des boues de vidange par Lit de Séchage à Ecoulement Non Saturé (LSENS). Journal de la Société Ouest-Africaine de Chimie, 29 : 1-10.

Memento Technique de l'Eau. 1989. Edition du cinquantenaire, $9^{\text {ème }}$ édition, Tome I, Degremont, 592p.

Mungray AK, Kumar P. 2008. Degradation of anionic surfactants during drying of UASBR sludges on sand drying beds. Journal of Environmental Management, 88(4) : 995-1002.

N'Diaye DA, Kankou OA, Namr IK. 2011. Evaluation des teneurs en coliformes fécaux par couplage avec des paramètres physicochimiques en ACP: cas des effluents de la STEP du périmètre maraicher de Sebkha, Nouakchott. Cameroon Journal of Experimental Biology, 07 (01) : 35-40.

Rodier J. 1996. Analyse de l'eau : eaux naturelles, eaux résiduaires, eau de mer (8ème édn). DUNOD : Paris ; 1384p. 